\title{
LA RESPONSABILIDAD JURÍDICA DE LOS PROGRAMADORES POR LOS DAÑOS CAUSADOS POR AGENTES AUTÓNOMOS
}

\author{
Alejandro Zornoza Somolinos \\ Universidad Carlos III de Madrid, Estudiante de Doctorado en Derecho Privado, \\ 100062467@alumnos.uc3m.es
}

\begin{abstract}
Resumen
El sistema de Derecho español no se elaboró para contemplar la presencia de agentes autónomos. Ante cualquier daño que éstos produzcan, los juristas nos vemos obligados a aplicar los mecanismos tradicionales de imputación de responsabilidad y riesgos $y$, en consecuencia, se concluye que el programador de un agente autónomo es el responsable único y absoluto de los daños producidos por el comportamiento autónomo del agente. Por insólito que parezca, esta realidad se ve especialmente agravada cuando el daño se ha debido al correcto funcionamiento del agente autónomo, ya que entre su comportamiento esperado y el daño producido, existe una relación de causalidad que impide la exculpación del programador.
\end{abstract}

Palabras Clave: Robótica, Inteligencia Artificial, Agente Autónomo, Derecho, Responsabilidad, Daños.

\section{APROXIMACIÓN A LOS REGÍMENES JURÍDICOS DE RESPONSABILIDAD CIVIL}

En el Derecho español podemos encontrar tres regímenes diferentes de responsabilidad civil extracontractual: la responsabilidad por los actos propios; la responsabilidad por los actos de aquellas personas de las que se debe responder; y la responsabilidad por los daños causados por las cosas que uno tiene bajo su guarda.

El primer grupo encuentra cobertura legal en el artículo 1902 del Código Civil (Cc), que indica que aquel que por su acción u omisión cause un daño a otro, y este daño se haya debido a su culpa o negligencia, está obligado a reparar el daño producido. Es lo que se conoce como principio de responsabilidad subjetiva por culpa del causante del daño.

El segundo grupo se encuentra contemplado en el artículo $1903 \mathrm{Cc}$, que comienza con una afirmación general indicando que la obligación de reparar el daño del artículo 1902 Cc no sólo es exigible para los actos propios, sino también para los de aquellas personas de quién se debe responder. A partir de ahí se realiza una enumeración de situaciones en las que aquellas personas de quién cabe esperar un comportamiento responsable en la vigilancia y cuidado de quiénes se encuentran a su cargo (culpa in vigilando), o de quiénes se les considera responsables de la delegación de tareas en otras personas (culpa in eligendo), deben responder por los daños que producen a quiénes debió vigilar o elegir con mayor diligencia. Así es que, por ejemplo, los padres son responsables de los daños que producen sus hijos, y los empresarios son responsables de los daños que producen sus empleados.

El tercer grupo se encuentra repartido entre los artículos 1906 a 1909 Cc, y de manera sucinta podemos reducirlo a que cuando el propietario de un bien, de un animal o de un terreno no ha cumplido con sus obligaciones de cuidado, vigilancia y/o mantenimiento, y dichos bienes, animales o terrenos generan un daño o perjuicio en otras personas, los propietarios negligentes están obligados a reparar dicho daño o perjuicio. Por citar algunos ejemplos, el propietario de una caldera que explota es el responsable de los daños producidos por la explosión; el dueño de un perro que muerde a una persona es el responsable de los daños que sufra esa persona; y el dueño de una finca en la que hay un árbol cuyas raíces terminan pasando a la finca vecina y desregularizan el terreno, será responsable de los perjuicios que produzca esa deformación.

En todos estos supuestos podemos saber a quién atribuirle la responsabilidad de los daños que se han producido y es lo que denominados responsabilidad subjetiva, porque el sujeto al que señalamos como responsable lo es bien por sus actos propios bien por su propia negligencia.

Sin embargo, existe una categoría especial de responsabilidad que se ha ido perfilando con los años, y es el denominado régimen de responsabilidad objetiva o de responsabilidad por los riesgos creados. En efecto, se parte de la idea de que una persona no sólo es responsable de los daños causados 
con motivo de sus acciones, sino que también es responsable de determinados actos en virtud del riesgo creado por el mero hecho de realizarlos. Un ejemplo claro y cotidiano es el de la conducción de automóviles: conducir es una actividad que genera un riesgo para otras personas, por lo que la mera posesión y utilización de un vehículo suponen por sí solas la existencia de ese riesgo, y por esta razón estamos obligados a tener un seguro de responsabilidad civil.

En cualquier caso, ya estemos ante un supuesto de responsabilidad objetiva o subjetiva, residen en la conciencia colectiva dos ideas respecto a la producción de un daño que debemos procurar que desaparezcan. La primera es que si existe una persona física a quién responsabilizar, es infinitamente más sencillo asumir que se ha sufrido un daño. La segunda es el convencimiento de que una vez resarcido económicamente un daño, éste desaparece. Esta objetivación del daño y de la responsabilidad a través del dinero está pensada para diluir en la sociedad humana las consecuencias de los daños causados por comportamientos humanos. Pero ¿qué ocurre si el daño no lo produce un ser humano?

El desarrollo de la inteligencia artificial (IA) ha hecho tambalear todos los sistemas legales de responsabilidad del mundo, empezando por el nuestro propio, ya que en el plano teórico asistimos a un desplazamiento del riesgo, y por tanto de la responsabilidad, que va desde el actor humano hacia el agente autónomo (AA).

Retomando el ejemplo de la conducción, si el conductor humano delega la tarea de conducir en su vehículo autónomo, el cual se supone capacitado para manejar el vehículo sin intervención humana, en caso de accidente, ¿existe base teórico-legal para exigir responsabilidad al conductor? Por sorprendente que pueda parecer, consideramos que la respuesta es no. Pero entonces, ¿quién resarcirá los daños?

\section{EL PROGRAMADOR DE AGENTES AUTÓNOMOS COMO RESPONSABLE CIVIL DE LOS DAÑOS}

Como regla general, el conductor de un vehículo es el responsable de los daños causados con dicho vehículo por los riesgos creados con la conducción [4]. Incluso si el co-piloto tomase el control del automóvil y provocase un accidente, el conductor seguiría siendo responsable (en este caso, responsable solidario, porque se trataría de una responsabilidad compartida) por dos razones: porque así lo determinan las normas de circulación, y por el hecho de no haber asegurado lo suficiente su actividad y haber permitido la injerencia de terceros.

Tanto es así que existen casos singulares en que los Tribunales, para poder exonerar al conductor y considerar responsable al co-piloto por haber accionado éste el freno de mano y haber provocado un accidente, tuvieron que considerar co-conductor al co-piloto para atribuirle la autoría del acto relevante [1].

Se produjo en esta ocasión lo que se denomina una transmisión del riesgo, es decir, el riesgo de la actividad lo asumió otra persona diferente al autor inicial.

Y lo mismo sucede si un conductor permite que la IA de su vehículo sea el responsable del manejo del mismo: en modo autónomo, el conductor deja de ser conductor para convertirse en no-conductor o, en el peor de los casos, co-conductor, transmitiendo así el riesgo de la actividad al AA. En un escenario ideal, en caso de accidente el AA y el no-conductor se convertirían en responsables solidarios, pero como el AA no es sino un programa informático, no es posible exigirle ningún tipo de responsabilidad.

Ante la pregunta quién paga en caso de un accidente producido por un vehículo autónomo, aceptando la premisa de que el conductor humano en realidad no conducía, el argumento impulsivo sería que, dado que el vehículo autónomo es propiedad del noconductor, se debería exigir del no-conductor la responsabilidad de los daños producidos por sus bienes, tal y cómo hemos visto que prevé el Código Civil.

Pero es aquí donde encontramos el elemento disruptivo con la doctrina tradicional: hasta ahora, los bienes de una persona no tenían la capacidad de tomar decisiones. En otras palabras; un objeto no podía dañar a una persona sino era por la culpa o negligencia de su propietario.

Esta falta de nexo de unión entre el daño producido por un AA y la no mediación de su propietario nos impide afirmar que el propietario/conductor de un vehículo autónomo es el responsable de los daños producidos autónomamente por su vehículo, tanto por ser injusto desde el punto de vista de la responsabilidad, como por ser una solución contraria a toda una batería de normas internacionales de protección de las personas.

Por tanto, debemos seguir reflexionando.

Para ello, en lugar de preguntarnos quién resarcirá el daño debemos preguntarnos por qué se ha producido el daño. Si la respuesta es por un error en la IA, la 
depuración de responsabilidades es sencilla: o bien es atribuible al productor por un defecto de fábrica, como sucede hoy en día con los problemas mecánicos, o bien es atribuible al propietario por una mala conservación, cómo también sucede hoy en día con los problemas mecánicos.

Pero, ¿y si el daño se produce por una desafortunada decisión del AA? Entonces el panorama legal se complica.

$\mathrm{Si}$, como consecuencia de una correcta programación, la entrada $\mathrm{A}$ ha dado como resultado la salida B, y esta salida ha sido la causante del daño, la lógica legal es exigir la responsabilidad a la persona que programó dicho protocolo.

En consecuencia, el programador que determinó cuál es el comportamiento esperado del vehículo autónomo es quién responderá, en última instancia, de los daños producidos por su diseño.

Este mismo discurso no sólo es aplicable a los vehículos autónomos, sino que vale para cualquier AA que produzca un daño. Imaginemos un robot asistencial que se mueve por una residencia de personas mayores. Durante un desplazamiento el robot trata de esquivar un obstáculo pero en su ejecución rompe una valiosa escultura. Si esquivar el obstáculo es el comportamiento esperado del AA, la responsabilidad del daño producido por su comportamiento esperado, ¿debería recaer sobre su programación?

Imaginemos también que empleamos un AA en un evento para que guíe a los visitantes, y durante su ruta una entrada determinada genera como salida que el robot debe detenerse, pero al no hacerlo en las condiciones adecuadas de seguridad alguien tropieza con él y se lesiona. El AA se comportó como era esperable ante determinado evento, pero su correcto comportamiento produjo un daño, por lo que ¿a quién deberíamos exigir responsabilidad?

La variedad de escenarios es prácticamente ilimitada, $\mathrm{y}$ en todos ellos resulta demasiado sencillo pensar que debería ser el propietario quién respondiese de los daños, pues al igual que sucede con un vehículo, al adquirir un AA se asumen los riesgos ineludibles que los AAAA crean en el ambiente. Pero lo cierto es que la relación de un propietario con su AA está más cerca a la relación de responsabilidad del progenitor que asume los daños que produce su hijo que de la relación de un conductor que provoca un accidente.

En estos casos existe un factor que no debemos olvidar: un niño debe ser educado en valores y comportamiento por sus progenitores, y ante un mal cumplimiento de estas obligaciones son ellos los responsables de no haber educado correctamente a su hijo, y por tanto deben resarcir los daños. Pero en el caso de un AA no existe más progenitor que el programador, ya que el propietario del vehículo autónomo lo adquiere para despreocuparse de la labor de conducir asumiendo que el vehículo conducirá correctamente, y el organizador de eventos que adquiere un AA guía asume que está capacitado para interactuar con el ambiente y evitar que las personas choquen contra él.

Desde el momento en que el comportamiento del AA, considerado un bien, un objeto, pasa a ser potencialmente impredecible, la responsabilidad de sus actos parece que recaerá, necesariamente, sobre el programador que estableció las bases de las predicciones.

\section{LOS LÍMITES EN LOS SEGUROS DE RESPONSABILIDAD PARA PROGRAMADORES DE AGENTES AUTÓNOMOS}

En pos de resolver todas las situaciones anteriores existe un argumento más sencillo si cabe: para cubrir los infortunios del vehículo autónomo, los del robot asistencial o los del robot guía, existen los seguros de responsabilidad civil de los propietarios, por lo que no sería necesario dirigir ninguna acción legal contra el programador. Pero la cuestión no es tan reducible.

No albergamos ninguna duda sobre que la existencia de un seguro favorece el resarcimiento y la objetivación de la responsabilidad, pero también es cierto que la presencia de un seguro siempre produce una des-socialización del ciudadano hacia las aseguradoras, considerando que la capacidad económica de éstas es ilimitado, y que por tanto están en condiciones de cubrir los daños cuando sea preciso.

Para poder asumir la importancia que tiene un seguro de responsabilidad en el ámbito jurídico debemos tener presentes dos cuestiones: la primera, que un daño no se hace desaparecer pagándolo. En estos casos lo único que se consigue es trasladarlo a otro plano, pues quién repara un daño sufre a su vez otro daño (en este caso, económico) por cubrir esa reparación. La segunda, que después de pagar una indemnización y/o reparación las aseguradoras no pueden regenerar los fondos perdidos con suficiente rapidez. En consecuencia, ante continuados siniestros, el único modo de poder obtener liquidez consiste o bien en aumentar el importe de las pólizas, trasladando el daño que intenta cubrir de un tomador 
al resto de clientes, o bien en reducir el tipo de siniestros que puede abarcar.

La razón por la que las aseguradoras limitan sus clientes a aquellos que cumplen determinados requisitos (en el caso de los conductores, los que tienen más de $\mathrm{X}$ años de carnet, por ejemplo) es para reducir las probabilidades de tener que pagar indemnizaciones. Se dice que gracias a la conducción autónoma se registrará un importante descenso de los accidentes de tráfico, y por tanto, se reducirán también las primas de los seguros. Esto no es necesariamente así. Si el responsable de un accidente en el que se ve involucrado un vehículo autónomo fuese siempre el propietario, ¿en qué condiciones las aseguradoras estarían dispuestas a cubrir a esa persona sabiendo que todas las veces, por pocas que fueran, debería asumir los costes del accidente?

Ya sea con primas altas o bajas, y recuperando la reflexión de que las aseguradoras deben regenerar fondos después de cada daño que resarcen, lo normal es que intenten desplazar la responsabilidad a otra persona para obtener de ella un resarcimiento, pues como decíamos, pagar un daño sólo genera otro daño en quién lo paga.

Así, llegamos a lo que se denominan situaciones de repetición de acciones. Es decir, si la persona que sufre el daño exige un resarcimiento económico y el seguro del propietario se ve obligado a pagar, éste puede después exigir al programador que le pague la cantidad que pagó al dañado (repetición de acción de indemnización), pues el seguro tuvo que cubrir económicamente los daños producidos por una programación incorrecta, o que siendo correcta, produjo un resultado lesivo.

Se trata en definitiva de desandar lo andado.

Una vez que nos encontramos en este estadio podemos pensar que, en cualquier caso, la empresa para la que trabaje el programador debe tener su propio seguro. Pero es que el argumento que estamos empleando no es cíclico, sino espiral; siempre tiene un inicio. De manera que si la aseguradora de la empresa para la que trabaja el programador debe hacer frente a las indemnizaciones por daños que sufran los propietarios de los vehículos autónomos que producen, las primas de estos seguros no podrán ser nunca bajas.

$\mathrm{Si}$ las primas son altas, los seguros por responsabilidad no quedan al alcance de todos, lo que resiente la profesión, la innovación y el desarrollo por motivos puramente económicos. Las empresas, a su vez, necesitarán asegurar fondos para cubrir sus pólizas en caso de accidente, por lo que repercuten el coste del seguro en el precio final del producto, produciendo tecnología artificialmente encarecida.

Como vemos, es relativamente sencillo encontrar soluciones que no satisfacen a nadie y que, por desgracia, todas terminan en el mismo punto: el programador es el origen del daño, especialmente si el producto funcionó cómo era esperable, pues ha sido justamente el comportamiento debido el causante de la lesión de una persona.

Como mero apunte anecdótico, el 31 de Mayo de 2016, el Committee on Legal Affairs [2] del Parlamento Europeo hizo público un Draft Report con recomendaciones dirigidas a la Comisión Europea en materia de robótica y Derecho Civil. Las Recomendaciones recogen el punto de vista de la institución que las emite y sugieren actuaciones a quien van dirigidas, pero no tienen ningún efecto legal vinculante como el que encontramos por ejemplo en las Directivas o los Reglamentos, que son de cumplimiento obligatorio para los Estados Miembros. Por esto, huelga decir que hay que darles la virtualidad normativa que tienen, que es prácticamente nula, aunque esto no significa que no que tengan la capacidad de influir profundamente.

Entre estas recomendaciones, el Committee sugiere que la responsabilidad por los daños causados por un AA recae sobre el programador en un principio, pero que dicha responsabilidad irá mermando y trasladándose gradualmente al propietario (al que en realidad identifican como teacher) en función de la capacidad de aprendizaje del agente, de manera que cuanto mayor sea la autonomía del agente, menor será la responsabilidad de las partes implicadas, y cuanto mayor sea la relación entre lo aprendido por el agente y la causa del daño, mayor será la responsabilidad del propietario. Ahora bien, matizan: no deben confundirse las habilidades "enseñadas" con las habilidades "auto-aprendidas". Con las habilidades enseñadas el Committee no está pensando en los supuestos de hecho que hemos visto antes, sino más bien en aquellos en los que los daños son producidos por AA cuando se les emplea como medios instrumentales para cometer un acto doloso, es decir, cuando el agente produce un daño por estar cumpliendo una orden del teacher.

En nuestra opinión, aunque esta diferenciación entre habilidades resulta pragmática, en realidad es un intento de disimular el discurso de todo lo hasta aquí expuesto: los fundamentos teóricos de la responsabilidad legal no pueden afrontar los daños producidor por un AA si no es responsabilizando al programador. 


\section{TÉCNICAS DE TRABAJOS PREPARATORIOS PARA REDUCIR LA RESPONSABILIDAD DE LOS PROGRAMADORES}

La regulación de una nueva realidad es siempre compleja y viene precedida de tres preguntas: quién la va a regular, cómo se va a regular, y cuándo se va a regular.

Las dos primeras cuestiones podemos responderlas con frases hechas, y tienen tantos adeptos como detractores: a) los expertos, y b) de la mejor forma posible, con el objetivo de mantener su atractivo y hacerla accesible a todo el mundo, aunque el Texto final no guste a todos. La tercera pregunta, en cambio, requiere algo más de caución.

Cuando aparece un nuevo uso de la tecnología también aparecen nuevos negocios jurídicos vinculados a ella. Un negocio jurídico es el acto por el cual se crean relaciones jurídicas que hacen nacer derechos y obligaciones para las personas implicadas. Por ejemplo, la compraventa es un negocio jurídico a través del cual el vendedor se obliga a entregar una cosa y el comprador se obliga a entregar el precio convenido.

Se dan situaciones en las que un negocio jurídico tiene una base tecnológica tan novedosa que no existen normas que lo regulen. En esos casos, se dice que ese negocio es alegal, lo que significa que no está prohibido, pero que tampoco está permitido. Lejos de que esta situación sea una panacea, un negocio jurídico sin regulación es un foco de inseguridad que a la larga se traduce en un deterioro de la confianza del ciudadano y de los inversores hacia la industria que se trata de impulsar, puesto que no se han previsto derechos ni obligaciones ni regímenes de responsabilidad específicos que aporten garantías.

Hoy, todo parece indicar que la robótica está llamada a correr esta suerte de alegalidad. Probablemente, asistiremos a unos años de desarrollo e implantación de la tecnología autónoma durante los cuales no existirán límites, ni derechos ni obligaciones concretas y propias de su sector. En el momento en que la regulación aparezca, como ya hemos presenciado en el caso de los Remotely Piloted Aircraft Systems (RPAS), veremos cómo el desarrollo de la industria y de la innovación se verá ralentizada, primero, por trámites administrativos, y después, por la falta de regulación jurídica de determinados escenarios, como hoy sucede ya con los riesgos para la privacidad que llevan implícitos los RPAS y que aun no se han solventado.
¿Debe la Inteligencia Artificial seguir evolucionando e implantándose en la sociedad civil hasta que se decida normar su situación? Lamentablemente sí, pero, afortunadamente, el desarrollo del llamado Derecho de las Nuevas Tecnologías y el venidero Derecho de la Robótica está permitiendo a los juristas desarrollar técnicas de estudio y de medida del impacto de la robótica en la esfera jurídica de los ciudadanos.

Entre estas técnicas se encuentra la llamada regulación desde el diseño (regulation by design) que consiste en incorporar al proceso de desarrollo tecnológico la consultoría jurídica profesional desde el primer estadio de un proyecto. De este modo, el jurista entra a formar parte del equipo de trabajo, realiza un análisis de las implicaciones legales del proyecto en cada fase y el diseño se puede ir modulando a medida que se desarrolla para evitar que el prototipo final resulte inservible, legalmente hablando.

La idea no debe desecharse ligeramente. Si valoramos que la teoría legal hoy permite señalar que algunos diseños de RPAS son incomerciables desde un punto de vista jurídico por suponer un elevado riesgo para la privacidad, ¿cuántas modificaciones no se habrían hecho durante el desarrollo para minimizar el impacto final? Lo mismo sucede en el campo de la IA desde que alcanzamos la conclusión (debatida) de que el programador de un AA puede ser el responsable de los daños que éste produzca.

Existe otra razón por la que no debemos oponernos directamente a la idea de que un jurista entre a formar parte de los costes de un proyecto, y es el Derecho Comparado. Éste consiste en estudiar una figura jurídica concreta a la luz de otra idéntica o similar que pertenece a un sistema de Derecho extranjero.

En efecto, en clara oposición a la universalidad del lenguaje de la Técnica se encuentra la virtualidad local del Derecho.

Ante la ausencia de una regulación europea a medio plazo, debemos tener presente que cada ordenamiento jurídico reaccionará de manera diversa ante los AAAA.

Así por ejemplo, la doctrina tradicional francesa contempla con total normalidad la denominada responsabilidad de las cosas, que si bien para un jurista español gráficamente tiene sentido, en el plano práctico se muestra muy inexacto. Esta responsabilidad plantea que los bienes realizan actividades que caen fuera del ámbito de control de su propietario, que es el único responsable. Desde la óptica de nuestro Derecho, esta situación sería 
equiparable a la elusión de una obligación de cuidado, aunque para la lógica francesa tiene un matiz claramente diferenciador que en España no alcanzamos a ver.

Ante este ejemplo de dispersión, y prediciendo que cada Estado aprobará su propia regulación en un principio y finalmente se unificará bajo el paraguas del Derecho Comunitario, la incertidumbre para el programador aumenta considerablemente a medio plazo:

Finalmente, nos queda por resolver la cuestión ética en las aplicaciones de la robótica.

Debemos adelantar que, ni tan siquiera en el ámbito práctico del Derecho Mercantil, es habitual realizar juicios morales sobre el impacto que pueda tener una nueva actividad económica. Esto se debe a que las game rules de los mercados son suficientemente claras y conocidas por todos.

En efecto, la ausencia continuada del debate éticojurídico en los planes de empresa y negocio actuales podrá tener dos consecuencias directas en la regulación desde el diseño: o bien que se ignore por completo la importancia de realizar un estudio ético de estas características, o bien que se normalice con el paso del tiempo hasta que llegue a ser una check task.

Pero, ¿tan importantes resultan las cuestiones éticas en la robótica aplicada? Lo cierto es que hasta que la sociedad se acostumbre a la presencia y uso de agentes autónomos, su creación y diseño deben considerarse un debate moral de primer nivel que se nos presentan en dos estadios.

Por un lado tenemos la cuestión sobre el uso ético de la robótica, que consiste en reducir los ingenios autónomos a herramientas, de manera que su finalidad ética se corresponde con el uso que se hace de ellas y la responsabilidad jurídica se regiría por los principios expuestos en este trabajo.

Por otro lado, tenemos la cuestión de la ética en la robótica, que consiste en determinar si, jurídicamente, la responsabilidad del programador puede modularse mediante la incorporación de valores éticos en los agentes autónomos.

Ambas cuestiones se cruzan en una maraña de preguntas ya habituales a estas alturas: ¿protagonizarán los robots la próxima revolución industrial a expensas de los trabajadores humanos? ¿Alcanzarán las máquinas la singularidad? ¿Debemos programar agentes autónomos con normas morales para protegernos de ellos?
Todas estas incógnitas caen de pleno en el ámbito de la Filosofía del Derecho, si bien desde nuestro punto de vista, ninguna puede solventarse únicamente con respuestas humanistas.

En nuestra opinión, este es un debate muerto en el que se tiende a confundir la moralidad con las normas cívicas, pues en definitiva la tecnología es neutral en valores: no distingue entre el bien y el mal.

Si un AA se detiene en una puerta para dejar salir antes de entrar, éste estaría cumpliendo una norma cívica, no una norma moral. Las primeras son fácilmente reproducibles y deben estar presentes en toda programación, pues si la tendencia es introducir AAAA en nuestra vida cotidiana, su actividad debe estar acomodada a nuestros usos sociales.

En cuanto a las segundas, en cuanto a las normas morales, pensemos en un AA de cirugía de tejidos blandos diseñado para operar en un quirófano. Si le trasladamos a una sala de torturas y se niega a rasgar la carne y ejecutar la acción, estaría cumpliendo un estándar moral, no uno cívico. Ello significaría que hemos sido capaces de comprender como funciona la moralidad en nuestro cerebro, y hemos podido reproducirla.

Logros tecnológicos a parte, el comportamiento moral (no cívico) se ve afectado por el Dilema del tranvía de Foot, en el que se presentan los conocidos escenarios de no-win [3]. Se trata de una serie de problemas morales en los que se debe decidir si se debe matar a una persona para salvar a varias. Por lo general, los problemas se resuelven con la fórmula de "la mejor opción posible es siempre la menos mala", lo que denota que la concepción de nuestra moral tiende a observar proporciones matemáticas que minimicen el número de daños.

¿Hasta cuándo podrá la IA desarrollarse sin trabas morales y legislativas? ¿Cuál es el régimen de responsabilidad aplicable? ¿Y el cuerpo normativo transitorio? En definitiva, ¿cómo puede todo un equipo de trabajo asegurar que su proyecto salvará todos los obstáculos legales previsibles si no es implicando en el proceso a un profesional de la legalidad?

\section{CONCLUSIONES}

Ante una realidad tan incipiente como la robótica, formular cualquier conclusión provisoria puede resultar aventurado, pero la exposición de hechos que hemos realizado en este trabajo no es caprichosa ni se trata de una reflexión aislada; es el resultado de una exégesis concienzuda de los textos doctrinales y normativos vigentes, los cuáles no están preparados 
para digerir la presencia de la IA y, en consecuencia, producen resultados injustos y desproporcionados.

Nuestro ordenamiento jurídico no contempla, ni tiene espacio para contemplar, un mecanismo de riesgo en las cosas que nos permita desvincular al programador de los resultados lesivos de los AAAA. Y tras la reciente comunicación del Committee, parece que tampoco en el espacio europeo se va a pugnar por un mecanismo de responsabilidad diferente, lo cual resulta excesivamente gravoso para la industria, en general, y para los programadores, en particular.

No podemos tender a la demonización de la tecnología ni a la criminalización preventiva de los programadores, pero tampoco existe base para hacer recaer sobre los propietarios de los agentes autónomos la responsabilidad compartida de una tecnología que ni comprenden ni controlan.

El recurso a los seguros de responsabilidad tampoco resulta sencillo, habida cuenta que estamos ante una actividad económica que se desarrolla en un mercado de libre competencia, por lo que, aunque su contratación resultase obligatoria como en el caso de los vehículos, impedir situaciones que pudiéramos considerar abusivas mediante limitaciones legales resultaría, cuánto menos, polémico.

Para que la robótica pueda desarrollarse de una manera equilibrada para programadores, inversores y usuarios, resulta perentorio elaborar una regulación adecuada, comenzando por determinar cuáles son los regímenes de responsabilidad. Pero para alcanzar un estadio normativo, primero deben existir unos fundamentos teórico-legales sobre los que erigir una normativa; es lo que se ha de venir a denominar Derecho de la Robótica. Esta disciplina no puede realizarse de manera aislada en Facultades de Derecho, sino que es necesario el trabajo conjunto de juristas y tecnólogos.

Mientras esto sucede, técnicas como la regulation by design ofrecen cierto alivio al impacto jurídico de la tecnología, incorporando al equipo de trabajo un jurista en los primeros estadios del proyecto. Si bien ante la actual falta de regulación expresa no se puede tender más que a una interpretación y adaptación de la normativa existente, he aquí una de las ventajas de estos procesos: las relaciones privadas reguladas en contratos o en protocolos de actuación son el marco perfecto para sentar las bases del Derecho de la Robótica, y dichas relaciones privadas nacen de los acuerdos elaborados por tecnólogos, inversores, ingenieros, programadores y juristas, sin intervención estatal. Es lo que se denomina autorregulación.

De un modo gráfico: cuando aparecieron las Leyes de Internet, los contratos electrónicos y negocios on line llevaban años utilizándose sin que existiesen normas para ellos. Fueron esos mismos contratos y negocios, que la industria ya había normalizado mediante su uso continuado, los que se usaron como base para elaborar tanto las Leyes nacionales como los Tratados Internacionales de Comercio Electrónico.

Incluso en el plano de la ética, podemos esperar a que el European Group on Ethics in Science and New Technologies (EGE) nos indique cuáles son las líneas morales que deben seguir nuestras máquinas, pero también podemos diseñar y defender nuestras propias corrientes de pensamiento para que les sirva de guía cuando llegue el momento.

Ahora, que parece que la robótica y la IA están preparadas para comenzar a interactuar con la sociedad civil de modo paulatino, es cuando deben comenzar los trabajos preparatorios conjuntos de base técnica y teórica que nos permitan elaborar un auténtico Derecho para la robótica. Nos encontramos ante la oportunidad de ocupar y modelar, con cierto antojo, un vacío legal que permanecerá libre durante un periodo de tiempo no superior a diez años. Se trata de aprovechar, en definitiva, la máxima de que no hay mejor manera de evitar los conflictos que anticipándose a ellos.

\section{Referencias}

[1] Anderson vs Territory Insurance Office Board (1999) NTSC 21, 128 NTR 16.

[2] Committee on Legal Affairs, Draft Report with recommendations to the Commission on Civil Law Rules on Robotics (2015/2103 (INL), de 31 de Mayo de 2016.

[3] Foot, P., The Problem of Abortion and the Doctrine of the Double Effect in Virtues and Vices, Oxford, Basil Blackwell, 1978.

[4] Real Decreto Legislativo 8/2004, de 29 de octubre, por el que se aprueba el texto refundido de la Ley sobre responsabilidad civil y seguro en la circulación de vehículos a motor, BOE núm. 267, de 05 de Noviembre de 2014. 